\title{
BIOSSÍNTESE E FUNÇÕES DAS LIPOXINAS NA RESOLUÇÃO DA INFLAMAÇÃO
}

\section{BIOSYNTHESIS AND FUNCTIONS OF LIPOXINS IN THE RESOLUTION OF INFLAMMATION}

\author{
Regina Sordi ${ }^{1}$, Octávio Menezes de Lima Jr², Jamil Assreuy². \\ 1 Departamento de Farmacologia, Universidade Federal de Santa Catarina, SC, Brasil. \\ E-mail: <reginadesordi@gmail.com> \\ 2 Laboratório de Farmacologia Aplicada, Farmanguinhos, Fundação Oswaldo Cruz, Rio \\ de Janeiro, Brasil.
}

Data de recebimento: $10 / 02 / 2012$

Data da aprovação: 22/05/2012

\section{RESUMO}

A resolução da inflamação é um processo endógeno ativo, e as lipoxinas são importantes mediadores desse processo. Os derivados do ácido araquidônico são biossintetizados principalmente nas mucosas e vasos sanguíneos e atuam principalmente em um receptor acoplado à proteína G (FPR2/ALX) expresso em uma variedade de tipos celulares. As lipoxinas exibem um grande repertório de ações pró-resolução, como a redução da infiltração de neutrófilos no local inflamado, diminuição da produção de superóxido e citocinas por granulócitos, ativação da fagocitose de microorganismos e de células apoptóticas por macrófagos, além de possuírem ações antinociceptivas diretas.

Palavras-chave: Lipoxinas. Resolução. Inflamação. FPR2/ALX. Lipoxigenase.

\begin{abstract}
The resolution of inflammation is an active endogenous process, in which lipoxins are important mediators. Lipoxins are mainly biosynthesized in the mucosa and blood vessels, and they and they activate a G-protein coupled receptor (FPR2/ALX) which is widely expressed in several cell types. Lipoxins have several pro-resolution actions, such as the reduction of the infiltration of neutrophil in the inflammatory site, the decrease in the production of superoxide and cytokines by granulocytes, the activation of phagocytosis of microorganisms and apoptotic cells by macrophages. In addition, lipoxins display direct antinociceptive effects.
\end{abstract}

Keywords: Lipoxins. Resolution. Inflamation. FPR2/ALX. Lipoxygenase.

\section{Introdução}

A resolução da inflamação, antes considerada um processo passivo, é hoje definida como um processo endógeno ativo que protege o hospedeiro de uma resposta inflamatória exacerbada (BANNENBERG et al., 2005; SERHAN et al., 2007). A inflamação é um dos principais componentes de muitas doenças da civilização moderna, como artrite, doenças cardiovasculares, câncer, obesidade, doença de Alzheimer e sepse, sendo que a inflamação crônica pode ser entendida, pelo menos em parte, como decorrente de um processo de resolução inadequado (HELGADOTTIR et al., 2004; KARP et al., 2004).

A resolução da inflamação é considerada um processo distinto de um processo anti-inflamatório. 
Isso porque, além de reduzir a infiltração de neutrófilos para os tecidos inflamados, as moléculas pró-resolução estimulam macrófagos dos locais inflamados a fazerem a retirada e o "clearance" de células apoptóticas e de micro-organismos, além de estimularem a atividade antimicrobiana de células da mucosa epitelial, tendo, portanto, um papel ativo e não apenas regulador (SCHWAB et al., 2007).

A resolução é acompanhada por uma troca ativa de mediadores predominantes em exsudatos inflamatórios. Inicialmente são gerados mediadores clássicos, como as prostaglandinas e leucotrienos, que ativam e amplificam os sinais cardinais da inflamação (dor, calor, rubor, edema). Então, as prostaglandinas E2 e D2 induzem a produção de enzimas que promovem gradualmente a síntese de mediadores que possuem atividade tanto anti-inflamatória quanto pró-resolutiva, como é o caso das lipoxinas, resolvinas, protectinas e maresinas. Essas famílias de moléculas endógenas pró-resolução não são imunossupressoras, mas atuam na resolução por ativar mecanismos específicos para promover a homeostasia (SERHAN et al., 2007, SERHAN et al., 2008a).

Tendo em vista a importância desses mediadores, o nosso objetivo é realizar uma breve discussão sobre as lipoxinas, desde sua biossíntese até o seu papel no processo da resolução da inflamação.

\section{Biossíntese de Lipoxinas}

As lipoxinas foram os primeiros mediadores reconhecidos com atividades tanto anti-inflamatória quanto pró-resolução e são pequenas moléculas derivadas de ácidos graxos poliinsaturados essenciais da dieta (ácido araquidônico), mais especificamente, do Ômega-6. Esses mediadores são biossintetizados pela ação sequencial das lipoxigenases e de outras enzimas (GODSON et al., 2000).

Os locais mais importantes de produção de lipoxinas são as mucosas e os vasos sanguíneos (Figura 1). Nas mucosas, as lipoxinas são geradas principalmente pelos neutrófilos a partir do 15-ácido-hidroxieicosatetraenoico (15-HETE), o qual é fornecido pelas células do epitélio (GRONERT et al., 1998; SCANNELL; MADERNA, 2006). Já nos vasos sanguíneos, a biossíntese envolve a oxigenação do ácido araquidônico pela 5-lipoxigenase em leucócitos e a liberação do intermediário leucotrieno $\mathrm{A}_{4}, \mathrm{O}$ qual é convertido em lipoxina pelas plaquetas através da atividade da 12-lipoxigenase (biossíntese transcelular) (ROMANO; SERHAN, 1992; SCANNELL; MADERNA, 2006).

Os neutrófilos também podem interagir nos locais inflamados com outras células e adquirir a habilidade de produzir lipoxinas, como outros leucócitos, células endoteliais, células epiteliais da mucosa e fibroblastos (WALLACE et al., 2003).

Figura 1 - Biossíntese de lipoxinas nas mucosas e vasos sanguíneos. AA: ácido araquidônico; PMN: células polimorfonucleares; 15-HETE: ácido hidroxieicosapentanoico; LO: lipoxigenase; LX: lipoxina. Adaptado de The Scientific World JOURNAL 2006; 6: 1555-1573

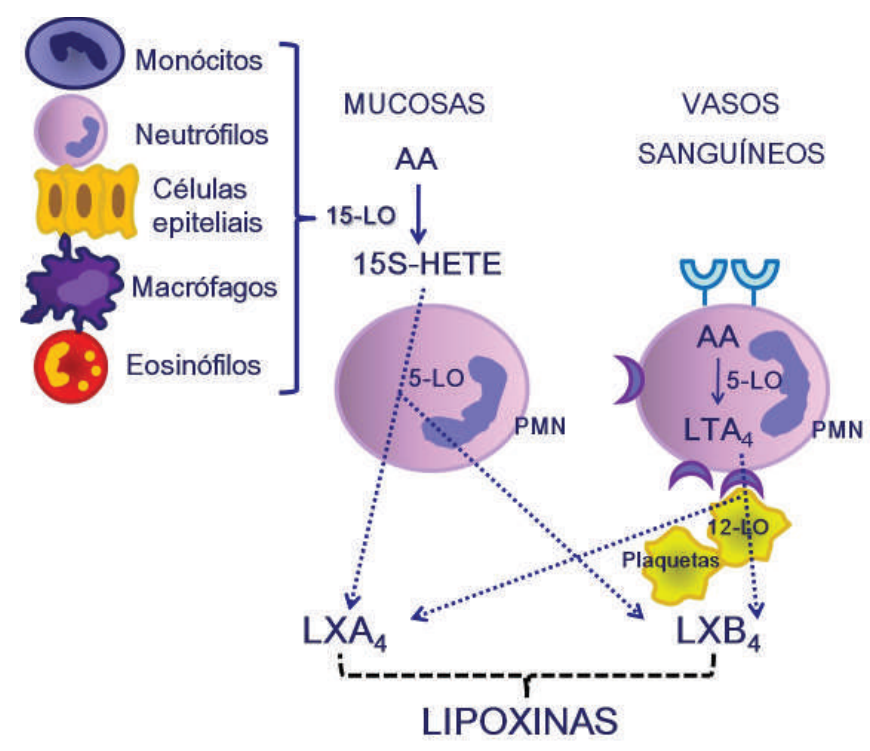

Um fato interessante é que além de a inibição da biossíntese de prostaglandinas pela aspirina ser um mecanismo importante para o efeito antitrombótico e anti-inflamatório do fármaco, a aspirina leva à formação endógena de epímeros de lipoxinas (15-epi-lipoxina) denominados ATLs (do inglês, Aspirin-Triggered Lipoxins) (CLÀRIA; SERHAN, 1995).

A acetilação da COX-2 pela aspirina bloqueia sua habilidade de biossíntese de prostaglandinas, porém ela permanece enzimaticamente ativa para produzir 15R-HETE a partir do ácido araquidônico, o qual é convertido pelos leucócitos em ATLs. Esses epímeros possuem as mesmas ações que as lipoxinas, entretanto são mais estáveis e assim possuem ação mais prolongada pois exibem atividade catalítica reduzida para inativação da enzima (SERHAN et al., 2008b). 
Dessa maneira, a aspirina, além de possuir ação anti-inflamatória, é um fármaco pró-resolução. Células que expressam COX-2, incluindo células do endotélio vascular, células epiteliais, macrófagos e neutrófilos estão envolvidos na produção de ATLs (Figura 2).

Figura 2 - Biossíntese de epímeros de lipoxinas pela aspirina. AA: ácido araquidônico; COX-2: ciclooxigenase-2; 15-HETE: ácido hidroxieicosapentanoico; PMN: células polimorfonucleares; LO: lipoxigenase; LX: lipoxina. Adaptado de Annu Rev Pathol. 2008; 3: 279-312

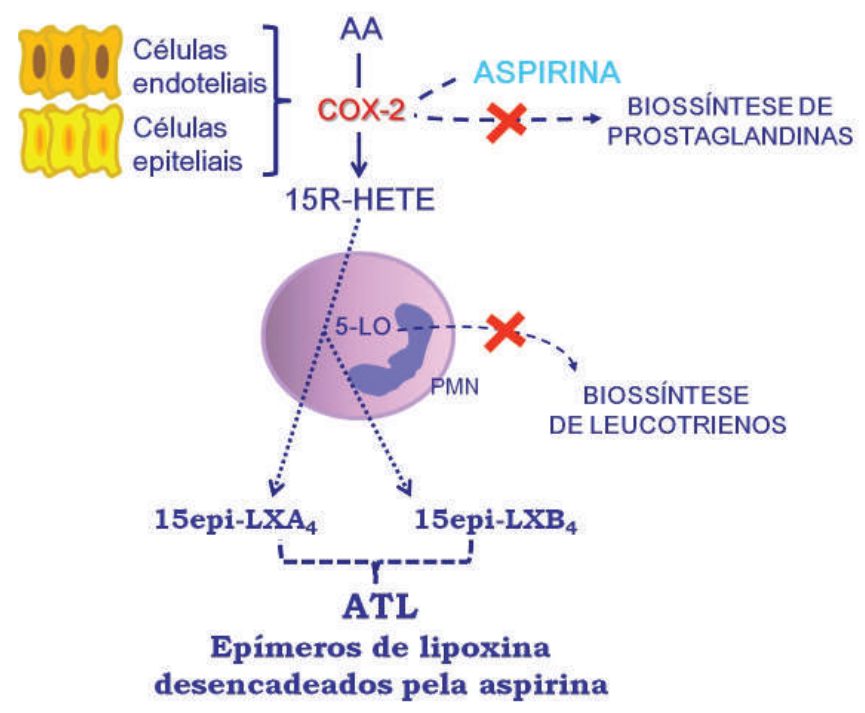

A produção de ATLs é aumentada em animais tratados com aspirina quando comparados a animais controle e também em humanos que ingerem baixas doses de aspirina (CHIANG et al., 2004). Quando desencadeadas pela aspirina, lipoxinas e 15-epi-lipoxinas estimulam a produção de óxido nítrico pelas células endoteliais, o qual impede a adesão de leucócitos nas células do endotélio vascular (PAUL-CLARK et al., 2004). Esse mecanismo pode ser particularmente relevante na resolução da inflamação local.

A aspirina também induz a expressão da heme oxigenase-1 (HO-1) de maneira independente de COX (GROSSER et al., 2003), oferecendo proteção contra insultos pró-oxidantes. A HO-1 é a primeira enzima envolvida no catabolismo do heme e o cliva para formar biliverdina e monóxido de carbono. As ATLs também são capazes de induzir a expressão da HO-1 pelas células endoteliais, um sistema importante na proteção tissular e também no processo anti- -inflamatório (NASCIMENTO-SILVA et al., 2005). A HO-1 parece ter um papel essencial nos efeitos anti-inflamatórios e de pró-resolução das ATLs (JIN et al., 2007).

\section{Receptores das Lipoxinas}

A lipoxina $\mathrm{A}_{4}\left(\mathrm{LXA}_{4}\right)$ e os epímeros de lipoxina (ATLs) atuam principalmente em um receptor acoplado à proteína $\mathrm{G}$ (GPCR) pertencente à família dos receptores FPR (do inglês, formyl peptide receptor), conhecidos pela importância na defesa do hospedeiro e na inflamação. Em humanos, há três receptores codificados por genes distintos: FPR1, FPR2/ALX e FPR3 (YE et al., 2009; DUFTON; PERRETTI, 2010).

O FPR2/ALX é o receptor ao qual a lipoxina se liga e é expresso em diversos tecidos, como baço e pulmão, e em distintos tipos celulares, como macrófagos, neutrófilos e microglia, tendo vias de sinalização específicas para cada tipo celular (CHIANG et al., 2006). Por exemplo, em neutrófilos, a interação LXA_-FPR2 impede a migração de neutrófilos, enquanto em monócitos, a interação da $\mathrm{LXA}_{4}$ ao receptor estimula a quimiotaxia de monócitos e outras respostas não flogísticas (MADDOX et al., 1997). Diferentemente dos GPCRs clássicos, que mobilizam cálcio intracelular para que ocorra quimiotaxia, as lipoxinas induzem mudanças na fosforilação de proteínas do citoesqueleto, resultando na detenção celular (REVILLE et al., 2006).

O FPR2/ALX é considerado um receptor promíscuo, possuindo ligantes peptídicos e lipídicos. A ligação de diferentes moléculas ao receptor ocorre com distinta afinidade e em diferentes locais de interação, facilitando a ativação de vias de sinalização distintas dependendo do tipo celular (BAE et al., 2003). Dentre os ligantes do FPR2/ALX estão a LXA $_{4}$, ATLs e análogos de ATLs, anexina-1, proteína amiloide, peptídeos do envelope do HIV, peptídeos antimicrobianos como LL37, entre outros (revisado em SCANNELL; MADERNA, 2006).

As lipoxinas também podem atuar como antagonistas parciais dos receptores cisteinil-leucotrienos (CysLT), sendo esse um potencial mecanismo através do qual as lipoxinas exercem seus efeitos anti-inflamatórios em algumas células e tecidos (GRONERT et al., 2001; MADERNA; GODSON, 2009). Um outro receptor da $\mathrm{LXA}_{4}$ é o receptor nuclear 
aril hidrocarboneto (AhR, do inglês, aryl hidrocarbon receptor), um fator de transcrição ativado por ligante. Foi demonstrado que a ativação do AhR pela $\mathrm{LXA}_{4}$ inibe a resposta imune inata de células dendríticas por aumentar a expressão do supressor de sinalização de citocinas-2 (SOCS-2, do inglês, suppressor of cytokine signalling 2), pertencente a uma família de proteínas que alteram e inibem respostas de citocinas.

Conforme citado anteriormente, a lipoxina e a anexina-1 (conhecida antigamente como lipocortina-1) compartilham o mesmo receptor FPR2/ALX (PERRETTI et al., 2002). A anexina-1 é uma proteína induzida por glicocorticoides bastante abundante no citoplasma de polimorfonucleares, monócitos e macrófagos. A administração de baixas doses de glicocorticoides em humanos (GOULDING et al., 1990) e roedores (PERRETTI; FLOWER, 1996) leva ao aumento do conteúdo proteico de anexina-1 nos leucócitos circulantes. Quando há ativação celular, por exemplo pela adesão de neutrófilos às células endoteliais, a anexina-1 é prontamente mobilizada na superfície da célula e secretada. Tanto a anexina-1 quanto seus peptídeos gerados enzimaticamente (por exemplo Ac2-26) são potentes inibidores da adesão e da migração de polimorfonucleares (PERRETTI; D'ACQUISTO, 2009).

Dessa forma, os glicocorticoides, além de suas propriedades anti-inflamatórias, também promovem resolução por estimular a retirada de neutrófilos apoptóticos por macrófagos (LIU et al., 1999), possivelmente por atuar através do fator anti-inflamatório endógeno anexina-1 (MADERNA et al., 2005) quanto por alterar o fenótipo de macrófagos durante a diferenciação por um mecanismo que envolve a reorganização de elementos do citoesqueleto (GILES et al., 2001).

\section{Ações das Lipoxinas}

Mediadores que possuem ações duplas por atuarem como anti-inflamatórios e pró-resolução, como as lipoxinas, exibem mecanismos de ação variados em modelos animais de doenças inflamatórias e promovem a resolução de inflamações orais, pulmonares, oculares, renais, dérmicas e gastrintestinais (SERHAN et al., 2008a).

No geral, as moléculas pró-resolução estimulam e aceleram a resolução por diversos mecanismos.
As lipoxinas reduzem a expressão de moléculas de adesão em leucócitos e células endoteliais, impedem a infiltração de neutrófilos e eosinófilos para o local inflamatório, reduzem a produção de superóxido e citocinas pelos neutrófilos, estimulam o recrutamento não flogístico de monócitos, ativam a fagocitose de micro-organismos e de células apoptóticas por macrófagos, aumentam a saída de fagócitos do local inflamado através do sistema linfático e estimulam a expressão de moléculas envolvidas na defesa antimicrobiana (Figura 3; CANNY et al., 2002; FILEP et al., 2005; CAMPBELL et al., 2007; SERHAN et al., 2008a,b).

Além desses efeitos na resolução da inflamação, a LXA 4 reduz a fibrose de órgãos, atuando diretamente no músculo liso vascular (CHIANG et al., 2006) e tem ações antinociceptivas diretas (SVENSSON et al., 2007).

Figura 3 - Algumas ações das lipoxinas. Adaptado de: $\mathrm{Br}$ J Pharmacol. 2009;158(4):947-959

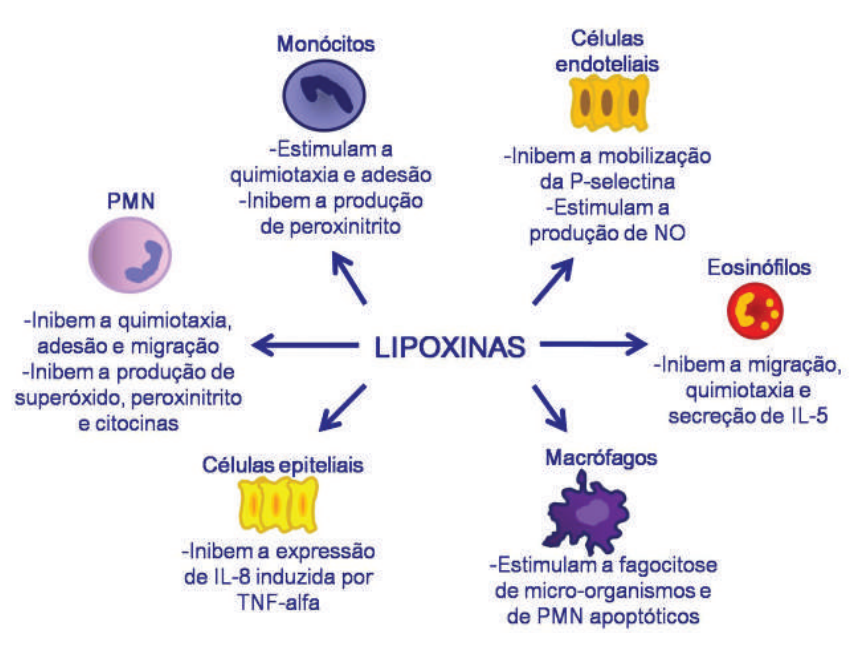

Um dado interessante é que patógenos também podem contribuir com a provisão de componentes necessários para a biossíntese de lipoxinas. Por exemplo, a bactéria Pseudomonas aeruginosa possui 15-lipoxigenase que converte o ácido araquidônico do hospedeiro em 15-HETE para a produção local de lipoxina (VANCE et al., 2004). Entretanto, a 15-lipoxigenase expressa por patógenos pode interagir com circuitos biossintéticos endógenos e gerar sinais que podem prejudicar a defesa imune do hospedeiro. Por exemplo, na infecção por Mycobacterium 
tuberculosis, o aumento de $\mathrm{LXA}_{4}$ endógena pelo hospedeiro regula negativamente a resposta protetora de células Th1 contra a infecção por micobactérias (BAFICA et al., 2005), indicando que o patógeno parece se beneficiar do ambiente anti-inflamatório proporcionado pela lipoxina. O estudo de Bafica e colaboradores (2005) demostrou que, na ausência da geração de $\mathrm{LXA}_{4}$ endógena, os camundongos tornaram-se mais resistentes à infecção, com redução do número de bactérias e aumento da taxa de sobrevida. Por outro lado, células do hospedeiro infectadas por Toxoplasma gondii, o qual também possui sua própria 15-lipoxigenase, produzem grandes quantidades de lipoxina (ALIBERTI et al., 2002; BANNENBERG et al., 2004), e a ausência de lipoxina endógena (utilizando-se animais 5-lipoxigenase) fez com que os camundongos sucumbissem rapidamente à infecção (ALIBERTI et al., 2002).

Analisando as duas infecções por $T$. gondii ou M. tuberculosis, os resultados mostram um efeito das lipoxinas produzidas endogenamente ora protetor, ora maléfico para o hospedeiro, podendo ser devido a diferenças na virulência dos patógenos em questão. O $T$. gondii replica-se rapidamente, e, para que a transmissão ocorra, o hospedeiro precisa sobreviver reduzindo o número de parasitas, os quais não devem, porém, ser totalmente eliminados. Para isso, as lipoxinas são induzidas para manter a resposta imune, mas não intensificá-la. Por outro lado, o M. tuberculosis possui crescimento lento e requer altas taxas de proliferação para que a transmissão possa ocorrer. Para isso são geradas lipoxinas para inibir a resposta imune, permitindo que o bacilo sobreviva. Em ambos os casos, a inibição de respostas pró-inflamatórias do tipo I via lipoxina fornece um ambiente favorável para transmissão e propagação do patógeno (ALIBERTI; BÁFICA, 2005).

\section{Conclusões}

As lipoxinas e os ATLs são derivados lipídicos que atuam ativamente no processo de resolução da inflamação, sendo cada vez mais estudadas e discutidas. Inúmeros trabalhos mostraram que a $\mathrm{LXA}_{4}$ e seus análogos têm importante impacto em uma variedade de estudos animais, sendo potencialmente interessantes para o uso na terapia de doenças inflamatórias humanas, como artrite, dermatite, asma, periodontite, entre outras.

\section{Referências}

ALIBERTI, J.; BAFICA, A. Anti-inflammatory pathways as a host evasion mechanism for pathogens. Prostaglandins Leukot Essent Fatty Acids, v.73, n.3-4, p.283-8, 2005.

; SERHAN, C.; SHER, A. Parasite-induced lipoxin A4 is an endogenous regulator of IL-12 production and immunopathology in Toxoplasma gondii infection. J. Exp. Med., v.196, p.1253-1262, 2002.

BAE, Y. S.; YI, H. J.; LEE, H. Y. Differential activation of formyl peptide receptor-like 1 by peptide ligands. J. Immunol., v.171, p.6807-6813, 2003.

BAFICA, A.; SCANGA, C. A.; SERHAN, C.; MACHADO, F.; WHITE, S.; SHER, A.; ALIBERTI, J. Host control of mycobacterium tuberculosis is regulated by 5 -lipoxygenasedependent lipoxin production. J Clin Invest., v.115, n.6, p.1601-6, 2005.

BANNENBERG, G. L.; ALIBERTI, J.; HONG, S.; SHER, A.; SERHAN, C. N. Exogenous pathogen and plant 15-lipoxygenase initiate endogenous lipoxin A4 biosynthesis. J. Exp. Med., v.199, p.515-523, 2004.

.; CHIANG, N.; ARIEL, A.; ARITA, M.; TJONAHEN, E.; GOTLINGER, K. H.; HONG, S.; SERHAN, C. N. Molecular circuits of resolution: formation and actions of resolvins and protectins. J. Immunol., v.174, p.4345-4355, 2005.

CAMPBELL, E. L.; LOUIS, N. A.; TOMASSETTI, S. E.; CANNY, G. O.; ARITA, M.; SERHAN, C. N.; COLGAN, S. P. Resolvin E1 promotes mucosal surface clearance of neutrophils: a new paradigm for inflammatory resolution. FASEB J., v.21, p.3162-3170, 2007.

CANNY, G.; LEVY, O.; FURUTA, G. T.; NARRAVULAALIPATI, S.; SISSON, R. B.; SERHAN, C. N.; COLGAN, S. P. Lipid mediator-induced expression of bactericidal/ permeability-increasing protein (BPI) in human mucosal epithelia. Proc. Natl Acad. Sci., USA, v.99, p.3902-3907, 2002.

CHIANG, N.; BERMUDEZ, E. A.; RIDKER, P. M.; HURWITZ, S.; SERHAN, C. N. Aspirin triggers antiinflammatory 15-epi-lipoxin A4 and inhibits thromboxane in a randomized human trial. Proc. Natl Acad. Sci., USA, v.101, p.15178-15183, 2004.

; SERHAN, C. N.; DAHLÉN, S. E.; DRAZEN, J. M.; HAY, D. W.; ROVATI, G. E.; SHIMIZU, T.; YOKOMIZO, T.; BRINK, C. The lipoxin receptor ALX: potent ligand-specific and stereoselective actions in vivo. Pharmacol. Rev., v.58, p.463-487, 2006. 
CLÀRIA, J.; SERHAN, C. N. Aspirin triggers previously undescribed bioactive eicosanoids by human endothelial cell-leukocyte interactions. Proc. Natl Acad. Sci., USA, v.92, p.9475-9479, 1995.

DUFTON, N.; PERRETTI, M. Therapeutic antiinflammatory potential of formyl-peptide receptor agonists. Pharmacol Ther., v.127, n.2, p.175-188, 2010.

FILEP, J. G.; KHREISS, T.; JÓZSEF, L. Lipoxins and aspirin-triggered lipoxins in neutrophil adhesion and signal transduction. Prostaglandins Leukot Essent Fatty Acids, v.73, n.3-4, p.257-62, 2005.

GILES, K. M.; ROSS, K.; ROSSI, A. G.; HOTCHIN, N. A.; HASLET,T. C.; DRANSFIELD, I. Glucocorticoid augmentation of macrophage capacity for phagocytosis of apoptotic cells is associated with reduced p130Cas expression, loss of paxillin/pyk2 phosphorylation, and high levels of active Rac. J. Immunol., v.167, p.976-986, 2001.

GODSON, C.; MITCHELL, S.; HARVEY, K.; PETASIS, N. A.; HOGG, N.; BRADY, H. R. Cutting edge: lipoxins rapidly stimulate nonphlogistic phagocytosis of apoptotic neutrophils by monocyte-derived macrophages. $\mathbf{J}$. Immunol., v.164, p.1663-1667, 2000.

GOULDING, N. J.; GODOLPHIN, J. L.; SHARLAND, P. R.; PEERS, S. H.; SAMPSON, M.; MADDISON, P. J.; FLOWER, R. J. Anti-inflammatory lipocortin 1 production by peripheral blood leucocytes in response to hydrocortisone. Lancet., v.335, p.1416-1418, 1990.

GRONERT, K.; GEWIRTZ, A.; MADARA, J. L.; SERHAN, C. N. Identification of a human enterocyte lipoxin A4 receptor that is regulated by IL-13 and IFN- $\gamma$ and inhibits TNF- $\alpha$-induced IL- 8 release. J. Exp. Med., v.187, p.12851294, 1998.

; MARTINSSON-NISKANEN, T.; RAVASI, S.; CHIANG, N.; SERHAN, C. N. Selectivity of recombinant human leukotriene $\mathrm{D}(4)$, leukotriene $\mathrm{B}(4)$, and lipoxin $\mathrm{A}(4)$ receptors with aspirin-triggered 15-epi-LXA(4) and regulation of vascular and inflammatory responses. Am $\mathbf{J}$ Pathol., v.158, n.1, p.3-9, 2001.

GROSSER, N.; ABATE, A.; OBERLE, S.; VREMAN, H. J.; DENNERY, P. A.; BECKER, J. C.; POHLE, T.; SEIDMAN, D. S.; SCHRÖDER, H. Heme oxygenase-1 induction may explain the antioxidant profile of aspirin. Biochem Biophys Res Commun., v.308, p.956-60, 2003.

HELGADOTTIR, A.; MANOLESCU, THORLEIFSSON, G.; GRETARSDOTTIR, S.; JONSDOTTIR, H.; THORSTEINSDOTTIR, U.; SAMANI, N. J.; GUDMUNDSSON, G.; GRANT, S. F.; THORGEIRSSON, G.; SVEINBJORNSDOTTIR, S.; VALDIMARSSON, E. M.; MATTHIASSON, S. E.; JOHANNSSON, H.; GUDMUNDSDOTTIR, O.; GURNEY, M. E.; SAINZ, J.; THORHALLSDOTTIR,
M.; ANDRESDOTTIR, M.; FRIGGE, M. L.; TOPOL, E. J.; KONG, A.; GUDNASON, V.; HAKONARSON, H.; GULCHER, J. R.; STEFANSSON, K. The gene encoding 5-lipoxygenase activating protein confers risk of myocardial infarction and stroke. Nat Genet., v.36, p.233-239, 2004.

JIN, S. W.; ZHANG, L.; LIAN, Q. Q.; LIU, D.; W. U. P.; YAO, S. L.; YE, D. Y. Posttreatment with aspirin-triggered lipoxin A4 analog attenuates lipopolysaccharide-induced acute lung injury in mice: the role of heme oxygenase-1. Anesth Analg., v.104, n.2, p.369-77, 2007.

KARP, C. L.; FLICK, L. M.; PARK, K. W.; SOFTIC, S.; GREER, T. M.; KELEDJIAN, R.; YANG, R.; UDDIN, J.; GUGGINO, W. B.; ATABANI, S. F.; BELKAID, Y.; XU, Y.; WHITSETT, J. A.; ACCURSO, F. J.; WILLS-KARP, M.; PETASIS, N. A. Defective lipoxin-mediated antiinflammatory activity in the cystic fibrosis airway. Nat Immunol., v.5, p.388-392, 2004.

LIU, Y.; COUSIN, J. M.; HUGHES, J.; VAN DAMME, J.; SECKL, J. R.; HASLETT, C.; DRANSFIELD, I.; SAVILL, J.; ROSSI, A. G. Glucocorticoids promote nonphlogistic phagocytosis of apoptotic leukocytes. J. Immunol., v.162, p.3639-3646, 1999.

MADERNA, P.; GODSON, C. Lipoxins: resolutionary road. Br J Pharmacol., v.158, n.4, p.947-59, 2009.

;; YONA, S.; PERRETTI, M.; GODSON, C. Modulation of phagocytosis of apoptotic neutrophils by supernatant from dexamethasone-treated macrophages and annexin derived peptide Ac(2-26). J. Immunol., v.174, p.3727-3733, 2005.

MADDOX, J. F.; HACHICHA, M.; TAKANO, T.; PETASIS, N. A.; FOKIN, V. V.; SERHAN, C. N. Lipoxin A4 stable analogs are potent mimetics that stimulate human monocytes and THP-1 cells via a G-protein linked lipoxin A4 receptor. J. Biol. Chem., v.272, p.6972-6978, 1997.

NASCIMENTO-SILVA, V.; ARRUDA, M. A.; BARJAFIDALGO, C.; VILLELA, C. G.; FIERRO, I. M. Novel lipid mediator aspirin-triggered lipoxin A4 induces heme oxygenase-1 in endothelial cells. Am. J. Physiol. Cell Physiol., v.289, C557-C563; 2005.

PAUL-CLARK, M. J.; VAN CAO, T. MORADIBIDHENDI, N.; COOPER, D.; GILROY, D. W. 5-epi-lipoxin A4-mediated induction of nitric oxide explains how aspirin inhibits acute inflammation. J. Exp. Med., v.200, p.69-78, 2004.

PERRETTI, M.; CHIANG, N.; LA, M.; FIERRO, I. M.; MARULLO, S.; GETTING, S. J.; SOLITO, E.; SERHAN, C. N. Endogenous lipid- and peptide-derived anti-inflammatory pathways generated with glucocorticoid and aspirin treatment activate the lipoxin A(4) receptor. Nat. Med., v.8, p.1296-1302, 2002.

.; D’ACQUISTO, F. Annexin A1 and glucocorticoids 
as effectors of the resolution of inflammation. Nat Rev Immunol., v.9, n.1, p.62-70, 2009.

.; FLOWER, R. J. Measurement of lipocortin 1 levels in murine peripheral blood leukocytes by flow cytometry: modulation by glucocorticoids and inflammation. $\mathbf{B r} \mathbf{J}$ Pharmacol., v.118, p.605-610, 1996.

REVILLE, K.; CREAN, J. K.; VIVERS, S.; DRANSFIELD, I.; GODSON, C. Lipoxin A4 redistributes myosin IIA and Cdc42 in macrophages: implications for phagocytosis of apoptotic leukocytes. J Immunol., v.176, n.3, p.1878-88, 2006.

ROMANO, M.; SERHAN, C. N. Lipoxin generation by permeabilized human platelets. Biochemistry, v.31, p.8269-8277, 1992.

SCANNELL, M.; MADERNA, P. Lipoxins and annexin-1: resolution of inflammation and regulation of phagocytosis of apoptotic cells. ScientificWorldJournal., v.6, n.6, p.1555-73, 2006.

SCHWAB, J. M.; CHIANG, N.; ARITA. M.; SERHAN, C. N. Resolvin E1 and protectin D1 activate inflammationresolution programmes. Nature, v.447, p.869-874, 2007.

SERHAN, C.N.; BRAIN, S.D.; BUCKLEY, C. D.; GILROY, D. W.; HASLETT, C.; O'NEILL, L. A.; PERRETTI, M.; ROSSI, A. G.; WALLACE, J. L. Resolution of inflammation: state of the art, definitions and terms. FASEB J., v.21, n.2, p.325-32, 2007.

.; CHIANG, N.; VAN DYKE, T. E. Resolving inflammation: dual anti-inflammatory and pro-resolution lipid mediators. Nat Rev Immunol., v.8, n.5, p.349-61, 2008a.

.; YACOUBIAN, S.; YANG, R. Anti-inflammatory and proresolving lipid mediators. Annu Rev Pathol., v.3, p.279-312, 2008b.

SVENSSON, C. I.; ZATTONI, M.; SERHAN, C. N. Lipoxins and aspirin-triggered lipoxin stop inflammatory pain processing. J. Exp. Med. ,v.204, p.245-252, 2007.

VANCE, R. E.; HONG, S.; GRONERT, K.; SERHAN, C. N.; MEKALANOS, J. J. The opportunistic pathogen Pseudomonas aeruginosa carries a novel secretable arachidonate 15-lipoxygenase. Proc. Natl Acad. Sci., USA, v.101, p.2135-2139, 2004.

WALLACE, J. L.; FIORUCCI, S. A magic bullet for mucosal protection. And aspirin is the trigger! Trends Pharmacol. Sci., v.24, p.323-326, 2003.

YE, R. D.; BOULAY, F.; WANG, J. M.; DAHLGREN, C.; GERARD, C.; PARMENTIER, M.; SERHAN, C. N.; MURPHY, P. M. International Union of Basic and Clinical Pharmacology. LXXIII. Nomenclature for the formyl peptide receptor (FPR) family. Pharmacol Rev., v.61, n.2, p.119-61, 2009. 